\title{
ADQUISICIÓN DE LOS RECURSOS GRAMATICALES DE REFERENCIA POR ESTUDIANTES DE ESPAÑOL COMO SEGUNDA LENGUA EN EL NIVEL AVANZADO
}

Silvia M. Vargas Víquez

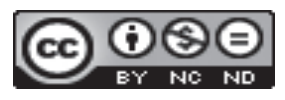

Esta obra está bajo una licencia Creative Commons Reconocimiento-No Comercial-Sin Obra Derivada 



\title{
ADQUISICIÓN DE LOS RECURSOS GRAMATICALES DE REFERENCIA POR ESTUDIANTES DE ESPAÑOL COMO SEGUNDA LENGUA EN EL NIVEL AVANZADO
}

\author{
ACQUISITION OF REFERENCE GRAMMAR RESOURCES BY SPANISH \\ AS A SECOND LANGUAGE ADVANCED STUDENTS
}

\author{
Silvia M. Vargas Víquez
}

\begin{abstract}
RESUMEN
Este estudio se enfocó en determinar si existe un patrón de adquisición de los recursos gramaticales de referencia en español. Los resultados revelan que se mantiene el mismo orden de preferencia de estos mecanismos en los diferentes niveles de proficiencia, incluso en el de avanzados. Persiste el uso del pronombre personal como primera opción de seguimiento de un participante en textos escritos por aprendices de español como segunda lengua.

Palabras clave: adquisición, segundas lenguas, referencia, cohesión.
\end{abstract}

\begin{abstract}
The findings of the investigation to determine the existence of an acquisition pattern of Spanish grammatical resources of reference reveal that the order of preference doesn't change from one level to another. This happens even at the advanced level, where the use of personal pronouns continues as participant follow-up first choice in texts written by second language Spanish learners.

Key words: acquisition, second language, reference and cohesion.
\end{abstract}

\section{Introducción}

La coherencia es un gran sistema que alberga diferentes mecanismos o estrategias lingüísticas que el hablante tiene a su alcance para lograr la unidad y la claridad de un texto. En palabras de Calsamiglia-Blancafort y Tusón-Valls (2007, p. 222), la coherencia incluye la cohesión; esta última se da toda vez que es necesario recurrir a un elemento del texto para interpretar otro o cuando se establecen conexiones entre las partes del discurso. Las relaciones

M.L. Silvia M. Vargas Víquez. Universidad de Costa Rica. Profesora de la Escuela de Filología, Lingüística y Literatura. Departamento de Lengua. Costa Rica.

Correo electrónico: silvarvi@yahoo.com

Recepción: 05- 11- 2013

Aceptación: 06- 01- 2014 
cohesivas se caracterizan por manifestarse concretamente en el texto, o sea que se pueden ver en operación en el discurso porque sus herramientas son: los conectores, la referencia, la elipsis y la sustitución (Halliday y Hasan, 1976, p. 4).

El lazo cohesivo que se investiga en el trabajo de Alvarado-Gutiérrez y Vargas-Víquez (2009) es la referencia, concretamente el mantenimiento de la referencia personal de la tercera persona singular por medio de recursos como el sustantivo común, el nombre propio, la pronominalización, la anáfora cero, los posesivos y los determinantes definidos e indefinidos. Los resultados del tercer nivel de proficiencia del corpus analizado indican que no se registran cambios significativos, en comparación con los principiantes e intermedios, sino que más bien persiste el uso no nativo de estos mecanismos referenciales.

A continuación, se resumen los principales fundamentos teóricos y metodológicos del estudio, los cuales aparecen más ampliamente expuestos en Vargas-Víquez (2012). Luego, se ofrecen los resultados del análisis de las veinte muestras del nivel avanzado de la investigación de Alvarado-Gutiérrez y Vargas-Víquez (2009).

\section{La referencia}

El concepto de referencia que se sigue en el estudio de Alvarado-Gutiérrez y VargasVíquez (2009) tiene como base la obra sobre la cohesión en inglés de M.A.K. Halliday y Ruquaiya Hasan (1976). Esta es definida como los recursos gramaticales que remiten a la identidad específica o definida de una entidad dentro de la situación comunicativa. Por lo tanto, se parte de la presunción de que la entidad a la que se hace referencia debe ser identificable.

La referencia es exofórica si la búsqueda de tal identidad debe hacerse fuera del texto y es endofórica si la información la brinda el texto mismo. Sólo la endófora es cohesiva. La exófora no contribuye a la integración de una sección con otra para que ambas formen parte del mismo texto, sino que relaciona elementos del texto con el contexto extralingüístico.

La relación que se establece entre las realizaciones referenciales y el antecedente es semántica; por tal razón no deben, necesariamente, coincidir en la forma gramatical.

Véase el siguiente ejemplo de una de las muestras de hablantes nativos (HN) del español en Alvarado-Gutiérrez y Vargas-Víquez (2009):

Mi papá $\underline{1}_{\text {se }}$ llama Wálter y desde muy pequeño ha trabajado 1 muy duro para poder darnos las necesidades básicas y nuestra profesión, lo cual representa para él l_lo fundamental para que, tanto mis hermanos como yo, podamos "defendernos" en la carrera de la vida. (HN1, L2)

Como puede observarse, se forma una cadena de referencia o series de realizaciones lingüísticas que remiten a un mismo referente: Mi papá $\leftarrow \varnothing$ ha trabajado $\leftarrow e ́ l$. Este tipo de referencia es la que Halliday y Hasan (1976, p. 3) llaman correferencial, porque tanto la anáfora cero (Ø) como él, tienen el mismo antecedente: Mi papá. Las cadenas correferenciales constituyen el foco de interés en el presente estudio.

Hay tres tipos de referencia: personal, demostrativa y comparativa. La personal funciona por medio de la categoría de persona, la información a la que remite es la identidad de los participantes en la situación comunicativa. La demostrativa localiza por medio de una escala de proximidad y la comparativa remite indirectamente estableciendo relaciones de igualdad o semejanza. La investigación se concentró en la referencia personal y en una de las realizaciones de la referencia demostrativa: el artículo definido. 
En español existen varios recursos o mecanismos para referir (Vargas-Víquez, 2012), por un lado, los que tienen que ver con el grupo o la frase nominal: primero, el grupo nominal sin modificadores (como podría ser el caso de los nombres propios); segundo, el grupo nominal con determinantes, en especial los artículos que indican si el participante ha sido mencionado antes (artículos definidos) o se está presentando por primera vez (artículos indefinidos); tercero, el grupo nominal con posesivos (que también funcionan como determinantes del grupo nominal, por lo cual rechazan la presencia simultánea de un artículo). Por otro lado, cuando se usan los pronombres para hacer una referencia se está en presencia de un fenómeno que se llama pronominalización. Se trata de una estrategia en la cual los pronombres se colocan en lugar de un sustantivo (Halliday, 2004, p. 554). Y por último, otro mecanismo referencial es la anáfora cero que se da cada vez que un verbo conjugado aparece sin un grupo nominal pleno o pronombre que lo acompañe, en este estudio se contabilizan las anáforas cero de tercera persona singular.

\section{Metodología}

En total se analizó un corpus de 60 muestras, escritas en español como segunda lengua (L2) por veinte hablantes nativos del inglés de cada nivel: principiante, intermedio y avanzado. La ubicación por niveles fue realizada por el Instituto de Lengua Española (ILE), lugar donde se recolectaron las muestras, a partir de los criterios de desempeño lingüístico establecidos por el American Council of the Teaching of Foreign Languages Inc. (2001). Cada informante describrió por escrito a los miembros de su familia.

Las 60 muestras de la investigación total fueron transcritas con todos los errores originales de puntuación y ortografía. En la transcripción de las muestras, los textos aparecen segmentados en unidades textuales que inician y terminan donde el informante utilizó un punto. Estas líneas fueron numeradas de forma consecutiva como se puede ver en el ejemplo que se ofrece más adelante.

Para identificar cada muestra se utilizó una inicial mayúscula que corresponde al nivel de dominio lingüístico del informante (P: Principiante, I: Intermedio, A: Avanzado) y un número correspondiente a la muestra. Además, en cada muestra se numeraron las líneas que corresponden al uso de punto y seguido o punto y aparte de cada informante. Por ejemplo, A2 corresponde a la muestra número dos del nivel avanzado y A2, L4 indica la línea 4 de esa misma muestra:

\section{Avanzado 2}

\section{Cadenas referenciales:}

(1) Mis padres murieron, mi padre ${ }_{1}$ hace 38 años y mi madre ${ }_{2}$ hace 7 años.

(2) Mi padre ${ }_{1}$ era un ingeniero civil y trabajó ${ }_{1}$ para el Departamento de Transportación del estado de California.

(3) Vivimos en California del Sur y nació en Los Angeles en 1944.

(4) A la edad de 4 años la familia se mudó a Redlands, 65 millas este de Los Angeles.

(5) Mi padre ${ }_{1}$ no le $e_{1}$ gustó la ciudad grande con mucha gente y tráfico. 
(6) Redlands es un lugar con muchos arboles de naranja.

(7) Mi madre ${ }_{2}$ asistía la iglesia evangelíca regularmente y traía ${ }_{2}$ nosotros los niños.

(8) Mi padre ${ }_{1}$ no assitía la iglesia regularmente.

(9) Tengo una hermana mayor y un hermano $_{5}$ menor.

(10) Mi hermana ${ }_{3}$ Enid se casó con Roberto ${ }_{4}$, un mecánico a la edad de 17; él 4 tenía 19 años.

(11) Ellos tienen dos hijas y seis nietos, la mayor ${ }_{0}$ se llama Kendall con 22 anõs.

(12) Mi hermano $_{5}$, Alan se casó con Shirley ${ }_{6}$.

(13) Despues de graduar de la universidad Alan $_{5}$ ha trabajado como un ingeniero electrico por 41 años.

(14) Alan $_{5}$ y Shirley tienen dos hijos y cuatro nietos, la mayor $_{0}$ se llama Raquel, con 15 años.

(15) Yo se casé con Gretchen .

(16) Tenemos dos hijos y tres nietos.

(17) Despues de 22 años de matrimonio separamos.

(18) He trabajado como un ministro de estudiantes universitarios, con varias responsabilidades en la iglesia y como un maestro de matemáticas y ciencia en varios colegios.

(19) Una cosa interesante es que para los últimos nueve años he enseñado en Latinoamería.

(20) Enseñe matemáticas, ciencia y la Biblia en ingles en colegios cristianos en Mexico, Guatemala y la Republica Dominicana.

(21) En julio pasado me jubiló.

Como puede verse en la muestra anterior (A2), se procedió a la identificación de las cadenas referenciales, en las cuales se les da seguimiento solamente a los referentes de tercera persona singular. Estos referentes están subindexados de forma consecutiva a partir del número uno. Todos los elementos lingüísticos que tienen el mismo número de subindexación corresponden a un mismo referente, con lo cual se puede seguir la cadena referencial a lo largo del texto. Véase en A2, la línea 7 donde se introduce a 'la madre 2 ' con sub- índice 2 y se le da seguimiento a su cadena referencial en esa misma línea con anáfora cero subindexada también con el número 2: 'traía 2 '.

El subíndice cero corresponde a aquellos referentes de tercera persona singular que sólo fueron mencionados una vez en el texto y por tal razón no fueron tomados en cuenta para el presente estudio, ya que no llegaron a conformar una cadena referencial.

Una vez transcrito y sistematizado el texto, toda la información se organizó en una tabla de clasificación como la siguiente: 
Tabla 1. Clasificación de las cadenas referenciales A2

\begin{tabular}{|c|c|c|c|}
\hline Línea & Realización & Clase & Referente \\
\hline 1 & padre $_{1}$ & $\mathrm{SC}$ & El papá \\
\hline 2 & padre $_{1}$ & $\mathrm{SC}$ & \\
\hline 2 & $\varnothing$ trabajó & A & \\
\hline 5 & padre $_{1}$ & $\mathrm{SC}$ & \\
\hline 5 & $\mathrm{le}_{1}$ & $\mathrm{P}$ & \\
\hline 8 & padre $_{1}$ & $\mathrm{SC}$ & \\
\hline 1 & madre $_{2}$ & $\mathrm{SC}$ & \\
\hline 7 & madre $_{2}$ & $\mathrm{SC}$ & La mamá \\
\hline 7 & $\varnothing$ traía $_{2}$ & A & \\
\hline 9 & una hermana $_{3}$ & SC/DIndef & La hermana \\
\hline 10 & hermana $_{3}$ & $\mathrm{SC}$ & \\
\hline 10 & Roberto $_{4}$ & NP & El cuñado \\
\hline 10 & $\mathrm{el}_{4}$ & $\mathrm{P}$ & \\
\hline 9 & un hermano 5 & SC/DIndef & El hermano \\
\hline 12 & hermano $_{5}$ & $\mathrm{SC}$ & \\
\hline 13 & $\operatorname{Alan}_{5}$ & NP & \\
\hline 14 & Alan $_{5}$ & NP & \\
\hline 12 & Shirley $_{6}$ & NP & La cuñada \\
\hline 14 & Shirley $_{6}$ & NP & \\
\hline
\end{tabular}

La primera columna corresponde a la línea del texto. La segunda se llama realizaciones y contiene las formas gramaticales que usó el informante en el texto. El símbolo Ø junto a los verbos conjugados en tercera persona singular corresponde a la anáfora cero. La tercera es la clase, ahí se clasifica cada forma de acuerdo con su categoría gramatical según sea sustantivo común (SC), nombre propio (NP), pronombre (P), anáfora cero (A), determinante posesivo (DPos), sustantivo común con determinante indefinido (SC-DIndef), sustantivo común con determinante definido (SC-DDef) y, por último, en la columna referente se indica la línea donde empieza una cadena referencial y se le da un nombre dependiendo del parentesco con el informante.

Acompañando a esta tabla de clasificación de las cadenas referenciales aparece una segunda tabla denominada Frecuencia de uso de las realizaciones según clase que precisamente especifica la cantidad de menciones de cada recurso referencial según su clase y el total de realizaciones en la muestra. Este ejemplo es de la muestra A2: 
Tabla 2. Frecuencia de uso de las realizaciones según clase

\begin{tabular}{|c|c|}
\hline Clase & Cantidad de veces \\
\hline SC & 8 \\
\hline NP & 5 \\
\hline P & 2 \\
\hline A & 2 \\
\hline DPos & 0 \\
\hline SC-DIndef. & 2 \\
\hline SC-DDef. & 0 \\
\hline Total de realizaciones en las cadenas analizadas & $\mathbf{1 9}$ \\
\hline
\end{tabular}

El mismo procedimiento se aplicó tanto al corpus de hablantes no nativos (HNN) como a las seis muestras de hablantes nativos (HN) del español.

En el estudio de Alvarado-Gutiérrez y Vargas-Víquez (2009) se determinó la frecuencia de aparición de los diferentes tipos de realización en cada nivel para así identificar el patrón de uso de los mecanismos referenciales que se analizaron. Posteriormente se compararon los tres niveles y se determinó que seguían el mismo patrón. Además, esos resultados fueron a su vez comparados con los datos obtenidos de las muestras de los hablantes nativos (HN). En este artículo se ofrecen solamente los resultados del análisis del nivel avanzado y, con el fin de compararlos, también se incluyen los resultados del grupo de control.

\subsection{Definiciones operativas}

Para efectos de la contabilización de las realizaciones referenciales de cada texto se tomaron en cuenta las siguientes especificaciones:

a. Sustantivo común (SC): Aquellas realizaciones que tienen como núcleo un sustantivo común singular y que sus pre y pos modificadores, si los tiene, no son determinantes posesivos de tercera persona, ni artículos definidos ni indefinidos.

b. Nombre propio (NP): Aquellas realizaciones que tienen como núcleo un nombre propio. Siguiendo a Fernández Leborans (1999, p.106, 107 y 110) los nombres propios que aparecen en estructuras del tipo: Mi hermana es Julia, Mi hermano Carlos, Mi mamá se llama Clara, no se considerarán referenciales. A pesar de tener un valor identificador y de que algunos estudiosos (Saunders, 1999) toman en cuenta todas las apariciones de estas formas; para efectos de este estudio y en estos casos particulares, los nombres propios son postmodificadores y, por lo tanto, aparecen al lado de formas que cargan un peso referencial más fuerte como son los núcleos de cada frase o grupo nominal y que son los que se contabilizan.

c. Pronombre (P): Se toman en cuenta sólo los pronombres personales de tercera persona singular.

d. Determinante posesivo (DPos): Se contabilizan en esta categoría sólo los posesivos de tercera persona, que son los que tienen una función referencial endofórica.

e. Anáfora cero (A): Toda vez que se encuentre un verbo conjugado en tercera persona singular, pero sin un grupo nominal ni un pronombre que lo acompañe, se considerará anáfora cero.

f. Sustantivo común con determinante indefinido (SCDIndef): Los sustantivos comunes singulares acompañados por artículos indefinidos. Se considerará un como artículo indefinido cuando introduzca por primera un participante en el discurso. 
g. Sustantivo común con determinante definido (SCDDef): Los sustantivos comunes singulares acompañados por artículos definidos.

\section{Grupo de control}

Para confirmar los usos nativos de estos recursos fue necesario analizar un pequeño corpus compuesto por seis muestras escritas por adultos costarricenses a quienes se les dio las mismas instrucciones que a los hablantes no nativos (HNN): describa a su familia: a sus padres y a sus hermanos. Estos fueron los resultados:

El análisis de las muestras del grupo de control permitió establecer cuáles son los recursos referenciales que los hablantes nativos utilizaron al seguir las mismas instrucciones de redacción que se les propusieron a los hablantes no nativos.

Cuadro 1. Frecuencia de uso de las realizaciones según su clase. Hablantes nativos.

Total de realizaciones: 287

\begin{tabular}{|c|c|c|c|c|c|c|c|c|}
\hline $\begin{array}{c}\text { Muestra/ } \\
\text { Clase }\end{array}$ & $\mathbf{1}$ & $\mathbf{2}$ & $\mathbf{3}$ & $\mathbf{4}$ & $\mathbf{5}$ & $\mathbf{6}$ & Total & $\boldsymbol{\%}$ \\
\hline A & 24 & 13 & 18 & 31 & 21 & 9 & $\mathbf{1 1 6}$ & $\mathbf{4 0 . 4 1}$ \\
\hline SC & 5 & 7 & 6 & 7 & 18 & 14 & $\mathbf{5 7}$ & $\mathbf{1 9 . 8 6}$ \\
\hline P & 15 & 6 & 8 & 9 & 14 & 11 & $\mathbf{6 3}$ & $\mathbf{2 1 . 9 5}$ \\
\hline DPos & 11 & 3 & 0 & 8 & 7 & 9 & $\mathbf{3 8}$ & $\mathbf{1 3 . 2 4}$ \\
\hline NP & 0 & 0 & 0 & 6 & 1 & 0 & $\mathbf{7}$ & $\mathbf{2 . 4 3}$ \\
\hline SC-DIndef & 0 & 1 & 0 & 0 & $\mathbf{0}$ & 2 & $\mathbf{3}$ & $\mathbf{1 . 0 4}$ \\
\hline SC-DDef & 0 & 0 & 0 & 2 & $\mathbf{0}$ & 1 & $\mathbf{3}$ & $\mathbf{1 . 0 4}$ \\
\hline Total & 55 & 30 & 32 & 63 & 61 & 46 & 287 & 100 \\
\hline
\end{tabular}

El mecanismo más utilizado por los HN es la anáfora cero, en el segundo lugar aparece el sustantivo común y en tercer lugar el pronombre y los determinantes posesivos de tercera persona singular. Lo cual da como resultado el siguiente orden para los nativos:

$$
\mathrm{A}>\mathrm{P}>\mathrm{SC}>\text { DPos }>\text { NP }>\text { SC }- \text { DIndef }>\text { SC-Ddef }
$$

Figura 1. Patrón de uso de los mecanismos referenciales.

\section{Hablantes Nativos}

Por lo tanto, se observa que en este grupo de control y para esta tarea descriptiva específica, dichos elementos formaron cadenas referenciales dentro del discurso y presentaron el siguiente orden: sustantivo común con determinante definido $\rightarrow$ sustantivo común con determinante indefinido $\rightarrow$ nombre propio $\rightarrow$ determinante posesivo $\rightarrow$ sustantivo común $\rightarrow$ pronombre (personal o posesivo) en tercera persona singular $\rightarrow$ anáfora cero. La anáfora cero es la que presenta mayor frecuencia de uso y el sustantivo común con determinante definido el que presenta menor frecuencia de uso.

\section{Resultados de las muestras del nivel avanzado}

Los estudiantes de nivel avanzado utilizaron el pronombre como mecanismo referencial predilecto al registrar un $34.14 \%$ del total de realizaciones. Le sigue el sustantivo común (generalmente aparece con posesivo de primera persona singular: mi madre, mi hermano, etc.) 
con un porcentaje de uso del $26.34 \%$. Ambos recursos junto con la anáfora cero concentraron el $80 \%$ de los mecanismos a los que recurrió este grupo para mantener la referencia.

Los otros mecanismos registran una menor frecuencia de uso donde el determinante posesivo (su, sus) fue el más utilizado (10.4\%), mientras que el nombre propio se contabilizó en un $6.34 \%$ de los casos y el SC-DIndef en un $3.41 \%$ y el SC-DDef en un $1.13 \%$.

Cuadro 2. Porcentaje de uso de cada tipo de realización según clase. Nivel Avanzado

\begin{tabular}{|c|c|}
\hline Clase & Porcentaje \\
\hline P & 34.14 \\
\hline SC & 26.34 \\
\hline A & 18.21 \\
\hline Dpos & 10.40 \\
\hline NP & 6.34 \\
\hline SC-DIndef & 3.41 \\
\hline SC-DDef & 1.13 \\
\hline TOTAL & $\mathbf{1 0 0 \%}$ \\
\hline
\end{tabular}

Con estos resultados se establece que los estudiantes de nivel avanzado presentan el siguiente orden en la frecuencia de uso de los mecanismos referenciales (Figura 2), siendo el primero el más usado y el último el menos usado, la contabilización de las realizaciones según su clase gramatical y el porcentaje se presentan en el Cuadro 3.

\section{$\mathrm{P}>\mathrm{SC}>\mathrm{A}>$ DPos $>\mathrm{NP}>\mathrm{SC}-$ DIndef $>$ Sc-DDef}

Figura 2. Patrón de uso de los mecanismos referenciales.

Nivel Avanzado

Cuadro 3. Frecuencia de uso de las realizaciones según su clase. Nivel Avanzado Total de realizaciones 615

\begin{tabular}{|c|c|c|c|c|c|c|c|c|}
\hline $\begin{array}{c}\text { Clase } \\
\text { Muestra }\end{array}$ & $\mathbf{P}$ & $\mathbf{S C}$ & $\mathbf{A}$ & DPos & NP & SC-DIndef & SC-DDef & Total \\
\hline $\mathbf{1}$ & 10 & 4 & 3 & 2 & 1 & 1 & 0 & $\mathbf{2 1}$ \\
\hline $\mathbf{2}$ & 2 & 8 & 2 & 0 & 5 & 2 & 0 & $\mathbf{1 9}$ \\
\hline $\mathbf{3}$ & 3 & 7 & 13 & 1 & 0 & 0 & 0 & $\mathbf{2 4}$ \\
\hline $\mathbf{4}$ & 30 & 9 & 10 & 8 & 0 & 5 & 0 & $\mathbf{6 2}$ \\
\hline $\mathbf{5}$ & 5 & 11 & 3 & 1 & 0 & 0 & 0 & $\mathbf{2 0}$ \\
\hline $\mathbf{6}$ & 6 & 8 & 9 & 5 & 4 & 1 & 1 & $\mathbf{3 4}$ \\
\hline $\mathbf{7}$ & 0 & 2 & 1 & 2 & 0 & 0 & 1 & $\mathbf{6}$ \\
\hline $\mathbf{8}$ & 1 & 14 & 3 & 1 & 0 & 1 & 0 & $\mathbf{2 0}$ \\
\hline $\mathbf{9}$ & 8 & 6 & 6 & 2 & 0 & 0 & 0 & $\mathbf{2 2}$ \\
\hline $\mathbf{1 0}$ & 7 & 4 & 1 & 5 & 0 & 0 & 0 & $\mathbf{1 7}$ \\
\hline $\mathbf{1 1}$ & 45 & 19 & 9 & 5 & 11 & 0 & 0 & $\mathbf{8 9}$ \\
\hline $\mathbf{1 2}$ & 12 & 16 & 12 & 3 & 7 & 2 & 0 & $\mathbf{5 2}$ \\
\hline $\mathbf{1 3}$ & 3 & 5 & 1 & 0 & 1 & 1 & 0 & $\mathbf{1 1}$ \\
\hline $\mathbf{1 4}$ & 11 & 5 & 1 & 5 & 1 & 1 & 0 & $\mathbf{2 4}$ \\
\hline $\mathbf{1 5}$ & 5 & 6 & 4 & 1 & 0 & 0 & 0 & $\mathbf{1 6}$ \\
\hline $\mathbf{1 6}$ & 13 & 9 & 9 & 3 & 0 & 2 & 2 & $\mathbf{3 8}$ \\
\hline $\mathbf{1 7}$ & 15 & 4 & 7 & 1 & 0 & 2 & 0 & $\mathbf{2 9}$ \\
\hline $\mathbf{1 8}$ & 23 & 16 & 9 & 12 & 5 & 1 & 1 & $\mathbf{6 7}$ \\
\hline $\mathbf{1 9}$ & 2 & 3 & 0 & 0 & 0 & 0 & 0 & $\mathbf{5}$ \\
\hline $\mathbf{2 0}$ & 9 & 6 & 9 & 7 & 4 & 2 & 2 & $\mathbf{3 9}$ \\
\hline Total & $\mathbf{2 1 0}$ & $\mathbf{1 6 2}$ & $\mathbf{1 1 2}$ & $\mathbf{6 4}$ & $\mathbf{3 9}$ & $\mathbf{2 1}$ & $\mathbf{7}$ & $\mathbf{6 1 5}$ \\
\hline
\end{tabular}




\subsection{Pronombre}

De los datos anteriores se desprenden las siguientes observaciones para cada clase de realización. Primeramente, se encontraron cadenas referenciales bastante largas como la siguiente de la muestra A4, donde al referente La madre se le da seguimiento principalmente con pronombres personales de sujeto, en este caso ella, y en menor cantidad los de complemento indirecto, le o les:

(9) Mi madre $_{4}$ tiene 55 años.

(10) Ella 4 vivo en California del Sur también.

(11) Ella $\underline{4}_{4}$ tiene estatura mediana y $\underline{\emptyset \text { tiene }}{ }_{4}$ pelo castaño.

(12) Ø tiene 4 mucha disciplina y es muy organizada con sus cosas.

(13) Ella ${ }_{4}$ es maestra de niños a una escuela privada.

(14) Ella ${ }_{4}$ especilista de estudiantes con incapacidades.

(15) Ella le $_{4}$ gusta hablar con sus amigos por internet. (A4, L9-15)

Esta manera de mantener la referencia se observa en muchas de las muestras de avanzados; otro ejemplo lo constituye A11, donde Andrew se sigue en una larga cadena referencial en la cual prevalece el uso de los pronombres personales. En comparación con las muestras del grupo de control, el hablante nativo prefiere la anáfora cero en este contexto de uso, o sea, cuando el referente ya ha sido introducido y es clara su identificación; por esa razón la pronominalización preferida por los aprendices resulta repetitiva y se convierte en una marca de uso extranjerizado del español.

(40) Andrew $_{3}$ vive en Nueva York ahora.

(41) $\mathrm{El}_{3}$ es actor pero también él ${ }_{3}$ trabaja en un restorante.

(42) Porque los papeles del teatro no gana mucho dinero para él $l_{3}$.

(43) Pienso que él $l_{3}$ es muy, muy divertido!

(44) $\mathrm{El}_{3}$ es alto, tiene los ojos azules (como mi padre, y yo).

(45) $\mathrm{El}_{3}$ es guapo.

(46) $\mathrm{El}_{3}$ se graduó de la Universidad de "East Carolina.

(47) Estoy contenta de que él $l_{3}$ este en Nueva York porque mucha gente le dijieron a él $_{3}$ que ellos creían que él $l_{3}$ no podría obtener un papel en los teatros en Nueva York.

(48) Pero ellos están equivocados porque el ${ }_{3}$ ganó 3 papeles y él 3 llegó allí hace un año.

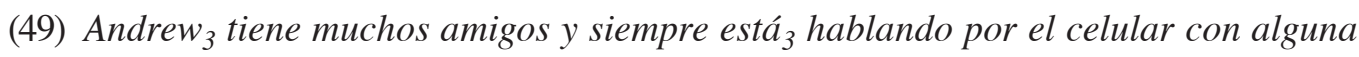
persona.

(50) Yo quiero una esposa para él ${ }_{3}$ que pueda entender la personalidad de él $l_{3}$ y tenga un buen humor, también quiera que él $l_{3}$ trabaje en un trabajo de teatro.

(51) Creo que en el futuro mi hermana 3 va a hacerse profesor del teatro en una universidad. 
(52) $\mathrm{A} \mathrm{él}_{3}$ le $_{3}$ gusta hacer sonrisas sobre las caras de otras personas.

(53) $\mathrm{El}_{3}$ es muy talentoso y creo que él $l_{3}$ está en la profesión que Dios le $e_{3}$ dio un talento a mi herman 3 para actuar.

(54) Andrew 3 y yo somos muy buenos amigos. (A11, L40-54)

La repetición de formas pronominales puede obedecer a la transferencia del idioma materno, que en este caso es el inglés; pues, según Payne (1992, p.42), en español las marcas verbales de persona son anafóricas y cuentan como única referencia del sujeto del verbo. Pero en inglés, las marcas verbales de persona solamente concuerdan con el sujeto del verbo que debe ser independientemente expresado (esto es, con un grupo nominal o un pronombre). Valga señalar que este uso referencial de los pronombres se observó en el corpus desde el nivel de principiantes; sin embargo, en este nivel de avanzados se rompe tímidamente en unas pocas muestras, como la A16, donde aparece el uso de anáfora cero (Líneas 3 y 4), y determinante posesivo como en las línea 7; pero sigue prevaleciendo la pronominalización (Líneas 3, 4 y 5):

(3) Mi mamá a $_{3}$ tiene sesenta y nueve años y trabaja 1 como cajera en un banco antes de retirarse.

(4) Ella 3 vive en Adamsville, Tennessee y disfruta 3 crecer muchas floras.

(5) Además, ella 3 le 3 gusta pintar.

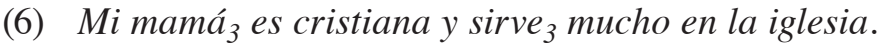

(7) $\mathrm{Su}_{3}$ esposo $_{1}$ es el dueño de una compañía de construcción. (A16, L3-7)

Además, del total de realizaciones pronominales con verbos de afección (31 en total) la mayoría hace un uso del pronombre de objeto indirecto cercano al nativo, en muchas incluso duplicado; lo que faltó en nueve de ellas fue la preposición $a$. Esto hace pensar que es una estructura que le indica al aprendiz las formas referenciales que debe colocar. Sin embargo, la adquisición total de este uso específico está en proceso, pues, al lado de ejemplos como los de las muestras A11, A14, y A20 donde parece haber adquisición, se pueden hallar otros como los de las muestra A17 y A18 que revelan que no la hay:

(6) $\underline{A ~ e ́ l}_{1} \underline{\text { le }}_{1}$ gustaba bailar y jugar golf.

(22) $\underline{\text { A ella }}_{2}$ le 2 gusta cocinar pero a ella 2 no le $\underline{2}_{2}$ gusta cuando otros mienten sobre cosas.

(52) $\underline{A ~ e ́ l ~}_{1} \underline{\text { le }}_{1}$ gusta hacer sonrisas sobre las caras de otras personas. (A11, L6, 22 y 52)

(18) $\underline{L e}_{3}$ gusta mucho pintar con óleo. (A14, L18)

(6) Le $\underline{L}_{1}$ gusta mucho las cultivar a los plantas y las flores. (A20, L6)

(10) $\underline{\text { A ella }}_{2} \underline{\text { le }}_{2}$ gusta cocinar y es 2 la cocinera mejor en todo el mundo (A20, L10)

(12) $\underline{E l l a}_{2} \underline{l e}_{2}$ gusta coser. (A17, L12)

(23) $Y \underline{e}_{6} \underline{l e}_{6}$ gusta cazar y pescar. (A18, L23)

(34) $\underline{E} \underline{l}_{1}$ también $\underline{l e}_{1}$ gusta jugar juegos de videos y hablar con sus $s_{1}$ amigos en la computadora (A18, L34) 


\subsection{Sustantivo común}

En el segundo lugar de frecuencia de uso entre los estudiantes avanzados está el sustantivo común (SC) y es el único mecanismo que está presente en todas las muestras de este nivel. Para efectos de este estudio un sustantivo común es una realización que tiene como núcleo un sustantivo común y que sus pre y pos modificadores, si los tiene, no son determinantes posesivos de tercera persona, ni artículos definidos, ni indefinidos. En otras palabras que aparezca sólo con el determinante posesivo de primera persona singular:

(5) Mi padre vive en Franklin, KY y no tiene $_{1}$ trabajo ahora. (A12, L5)

(11) Mi $\underline{\text { madre }}_{2}$ se llama Amy y tiene 244 años. (A12, L11)

Cabe resaltar que, sumando todos los sustantivos comunes como una solo categoría (30.88\%), alcanzan un porcentaje cercano al del pronombre (34.14\%), lo cual parece indicar que el estudiante alterna estas dos formas para mantener la referencia y que recurre a estos mecanismos respondiendo a la necesidad de que no falte nunca la marca referencial explícita que acompañe a toda forma verbal conjugada, como la transferencia del inglés se lo indica. Estos dos marcadores referenciales abarcan el $65 \%$ del total de realizaciones del nivel.

\subsection{Anáfora cero}

En tercer lugar aparece la anáfora cero (A) que tiene 112 realizaciones, lo que representa un $18.21 \%$ del total de realizaciones. En este grupo de muestras se pueden observar dos indicios de que la adquisición de este recurso ha avanzado un poco respecto a los otros niveles:

Por un lado, cada una de las muestras de este nivel presenta anáfora cero al menos una vez, excepto la número 19 donde del todo no aparece. La mayor parte de las veces aparece poco, lo cual puede reflejar inseguridad y una necesidad de conocer los contextos de uso de este recurso; pues, de las 112 realizaciones de anáfora cerco, el 49\% (o sea, 55) aparecieron precedidas por la conjunción $y$, lo que significa que ese contexto le provee el aprendiz la seguridad necesaria para utilizar esta forma referencial, como se observa en el siguiente ejemplo:

(4) Mi hermana 2 es casada y Ø tiene 24 hijos.

(5) También es $e_{2}$ ama de casa y Ø vive 2 cerca de mis padres. (A15, L4 y L5)

Por otro lado, en la muestra A3 la anáfora cero fue el recurso referencial más frecuente, con 13 realizaciones de un total de 23, lo cual la acerca al patrón nativo. Véase la muestra y su correspondiente cuadro de frecuencia de uso de las realizaciones según clase (Cuadro 4):

\section{Avanzado 3}

(25) Tengo una familia muy buena, gracias a Dios.

(2) Todos son cristianos, y por eso estoy muy agradecida.

(3) Mi mamá ${ }_{1}$ creció en una granja en el estado de Minnesota, que está muy norte en los EE.UU.

(4) Ella ${ }_{1}$ es enfermera y trabaja ${ }_{1}$ en un hospital con los espué nacidos y sus mamas. 
(5) Mi papá 2 también es de Minnesota, pero de la ciudad de San Pablo, que es la capital del estado.

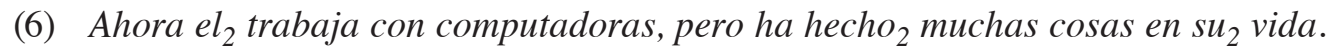

(25) $\mathrm{Iba}_{2}$ a ser pastor, psicólogo y constructor de casas (y realmente construyó ${ }_{2}$ la casa donde crecí.)

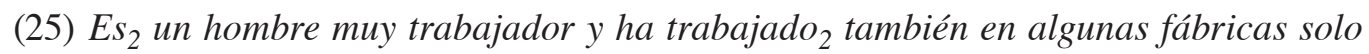
para poder pagar todos los gastos de la vida.

(25) Mis papas siempre han tenido expectativas muy altas para mi hermana 3 yo.

(10) Nos enseñaron la Biblia, como trabajar, el valor de una educación buena y amplia, etc.

(11) Mi hermana 3 se llama Kara, y es 3 menor que yo.

(12) Siempre era $\mathrm{en}_{3}$ mermanita y así siempre sera $\mathrm{s}_{3}$, creo.

(13) Pero ya se casó ${ }_{3}$ en agosto de este año, y compró ${ }_{3}$ una casa en Minneapolis antes de eso.

(14) Supongo que ya es 3 adulta también.

(15) Nosotros tenemos una relación muy buena ahora, pero antes, espuésos muchísimo.

(16) No puedo hablar de mi familia sin hablar de mi esposo 4 y mis hijos.

(17) $\mathrm{Mi}_{\text {esposo }}$ se llama Jeff, y es $_{4}$ muy bueno, claro.

(18) Nos conocimos en la universidad de Bethel que está en MN también.

(19) Aunque el 4 originalmente es de Colorado.

(20) Nos casamos durante el verano espués de graduarnos.

(21) Nos mudamos a California donde habíamos vivido por 8 años antes de vernir a Costa Rica.

(22) Durante este tiempo tuvimos dos hijos.

(23) Olivia $_{0}$ ya tiene años, y nuestro hijo ${ }_{0}$, Sawyer, tiene 2.

(24) Mis hijos son tan preciosos, y mi esposo 4 también.

(25) Dios me ha bendecido mucho en esta vida.

Cuadro 4. Frecuencia de uso de las realizaciones según clase Muestra A3

\begin{tabular}{|c|c|}
\hline Clase & Cantidad de veces \\
\hline SC & 7 \\
\hline NP & 0 \\
\hline P & 2 \\
\hline A & 13 \\
\hline DPos & 1 \\
\hline SC-DIndef. & 0 \\
\hline SC-DDef. & 0 \\
\hline Total de realizaciones en las cadenas analizadas & $\mathbf{2 3}$ \\
\hline
\end{tabular}


Sin embargo, y a pesar de estos indicios de un acercamiento al uso nativo, los resultados generales del nivel arrojaron que la frecuencia de uso de la anáfora cero no se equiparó a la del grupo de control y tampoco se marcó un cambio en el patrón de preferencia; pues, la pronominalización y el sustantivo común continúan registrando un porcentaje muy alto (como puede verse en los Cuadros 2 y 3); por lo tanto, este mecanismo no ha sido plenamente adquirido por los estudiantes de nivel avanzado

\subsection{Determinante posesivo}

Se usan 64 determinantes posesivos para un $10.4 \%$ en la frecuencia de uso. Resulta interesante que 17 de los 20 informantes de este nivel usaron este mecanismo. La mayoría lo usó menos de cinco veces, pero hay muestras como la A20 con 7, la A4 con 8 y la A18 con 12 y lo usan como los hablantes nativos, pues no aparece combinado con otros determinantes; lo cual podría deberse a que los contextos de uso de los posesivos son similares en inglés. Estos son algunos ejemplos:

(8) Ella 2 tenía trabajos a veces pero por mucho vivo a ella ${ }_{2}$, cuidaba $\underline{s u s}_{2}$ hijos y $\underline{s u}_{2}$ casa. (A20, L8)

(8) Cada julio su $_{1}$ familia fueron a la playa de Mrytte Norte en Carolina de Sur por todo el mes.

(9) $\underline{E}_{l}$ aprendió el baile de "Sag” y como jugaba ${ }_{1}$ golf alli durante $\underline{s u}_{1}$ adolence.

(10) Durante $\underline{s u}_{1}$ vida él $\underline{~}_{1}$ ganó muchos primios en las competencias de golf y también en las competencias del baile "SAG". (A11, L8-10)

\subsection{Sustantivo común con determinante indefinido}

Este mecanismo se usó en 15 ocasiones, lo que representa el 3.41\% del total. Dicho porcentaje podría considerarse bajo, pero no si se relaciona con el contexto de uso y con la tarea solicitada.

En el caso del contexto de aparición, los determinantes indefinidos suelen introducir un participante; lo cual reduce el uso de este recurso, pues generalmente en un texto corto cada participante va a ser presentado únicamente una vez.

\section{(1) Tengo un hermano mayor de mi. (A1, L1)}

Por otro lado, en el caso del tipo de tarea asignada, la descripción de la propia familia permite presentar a cada participante mediante el uso del determinante posesivo mi: mi mamá, mi hermana, etc. En la mayoría de los casos, no solo en este nivel sino en el corpus en general, fue necesario el uso del determinante indefinido sólo cuando los participantes se presentaron por primera vez y en oraciones transitivas con el verbo tener.

\subsection{Sustantivos comunes con determinante definido:}

Aparecen cinco casos de sustantivos comunes con determinante definido que son el $1.18 \%$ del total. Este porcentaje también puede haber sido el resultado del tipo de texto que se pidió que escribieran: descripción de su familia. Tal descripción estimula el uso de los nombres 
propios o de grupos nominales con determinantes posesivos de primera persona singular: $m i$ papá, mi mamá, que fueron contabilizados como sustantivos comunes (SC), en detrimento de la aparición de grupos nominales definidos. Véase el siguiente ejemplo de la muestra A6.

(1) Tengo cinco hermanos y dos hermanas.

(2) Yo soy el sexto con 2 hermanos menores.

(3) Yo nací y crecí en el estado de Nueva York y fui a universidad en Ohia cuando gradué de high school.

(4) Mi hermana $\underline{1}_{\text {mayor }}$ (Brenda) ya tiene 58 años y vive ${ }_{1}$ en Sur Carolina con su esposo $_{0}$.

(5) Ellos tienen 5 hijos y 6 nietos.

(6) Mi hermano $_{2}$ Don tiene 57 años y vive ${ }_{2}$ en Florida con su $_{2}$ esposa $a_{0}$ Sue y ellos, tienen 2 hijos y 3 nietos.

(7) Mi hermano $_{4}$ Roger tambien vive en Florida y el 4 y su esposa $_{0}$ (Barb) tienen 5 hijos y 5 nietos.

(8) El $_{4}$ tiene 56 años.

(9) Mi hermana ${ }_{5}$ Janette tiene 55 años y es ${ }_{5}$ soltera.

(10) Ella ${ }_{5}$ vive en el estado de Ohio.

(11) Mi hermano $_{6} \underline{L a v v y}_{\text {tiene }} 54$ años y vive ${ }_{6}$ en Nashville, Tennesse con su esposa $_{0}$ Brenda.

(12) Ellos tienen 1 hija 0 , todavía, 0 nietos.

(13) Mi hermana ${ }_{7}$ Gavy tiene 47 años y vive ${ }_{7}$ en Nueva York con su esposa $_{0}$ Cindy (A6, L1-13)

\section{Conclusiones}

Por una parte, en cuanto al número de mecanismos usados por muestra, no se puede establecer una relación entre el número de realizaciones y la diversidad de mecanismos utilizados. Por ejemplo, la muestra A11 es la que presenta más realizaciones (89 en total), pero no tiene ni sustantivos con determinante indefinido (SC-DIndef), ni sustantivos con determinante definido (SC-DDef). Sin embargo, en las muestras A16 y A20, a pesar de presentar menos realizaciones (36 y 39 respectivamente), figuran todos los tipos de mecanismos que se toman en cuenta en este estudio. Asimismo, el número de realizaciones no determina el dominio de estos recursos referenciales porque muchas veces se forman largas cadenas de pronominalización, lo que hace que el texto se aleje del uso nativo. En este mismo sentido, tampoco la cantidad indica si la tarea descriptiva se logra o no. Por ejemplo, tenemos la muestra A11 con 89 realizaciones, pero también la muestra A19 con 5 realizaciones. Sin embargo, la muestra A19 aún con una sola y muy corta cadena referencial, cumple con la tarea descriptiva que se solicitó en el instrumento. 
Por otra parte, los contextos de uso que exigen la aparición de ciertas formas referenciales por encima de otras, pueden darle al aprendiz mayor seguridad para usarlos. Se pudieron observar dos en especial. Primero, los pronombres personales de objeto indirecto deben aparecer con verbos de afección que exigen, además, la presencia de la preposición $a$ para la duplicación del complemento indirecto. Del total de realizaciones de este tipo (31) en la mayoría se usó el pronombre como lo hacen los HN, en muchas incluso reduplicado; lo que faltó en nueve de ellas fue la preposición $a$. Esto hace pensar que es una estructura que señala al aprendiz la forma que debe aparecer y eso contribuye para que lo haga con mayor seguridad. Segundo, 112 realizaciones de anáfora cerco, el 49\% (o sea, 55) aparecieron precedidas por la conjunción $y$, lo cual significa que este es otro contexto que le provee al aprendiz la información que necesita para colocar el mecanismo referencia adecuado.

En cuanto al patrón de frecuencia, contrario a lo que se podría haber pensado, no hay un cambio en el orden de la preferencia de uso de los mecanismos referenciales que lo acerquen al uso nativo; más bien persiste el patrón que apareció desde el nivel principiante.

$$
\mathrm{P}>\mathrm{SC}>\mathrm{A}>\mathrm{DPos}>\mathrm{NP}>\mathrm{SC}-\mathrm{D} \text { Indef }>\text { SC-DDef }
$$

En suma, dichos resultados revelan que no hay adquisición de los mecanismos de referencia al término de un año de estudio intensivo de español. Por lo tanto, es necesario incorporar este tema como un contenido en los programas de gramática de los institutos de español como segunda lengua; pues la enseñanza gramatical beneficia al aprendiz de diversas formas (Thornbury, 1999), a saber: funciona como una 'máquina para hacer nuevas oraciones', desarrolla la habilidad de evitar ambigüedades, evita la fosilización, funciona como un organizador anticipado de las nuevas estructuras, contribuye a organizar el lenguaje en categorías, las reglas muchas veces resultan orientadoras, llena las expectativas de los estudiantes de aprender de forma sistemática. De esta manera, es muy posible que si los profesores de español como segunda lengua son conscientes de esta deficiencia en la adquisición de mecanismos referenciales y de los beneficios que produce la enseñanza explícita de estas formas gramaticales y sus contextos de aparición, se podría acelerar su incorporación en la producción de los aprendices.

\section{Bibliografía}

American Council on the Teaching of Foreign Languages Inc. (ACTFL). (2001). Criterios de Proficiencia - Escritura. http://www.actfl.org/ [Consulta 15 de abril de 2009].

Alvarado-Gutiérrez, M. y Vargas-Víquez, S. (2009). Adquisición de los mecanismos de referencia por estudiantes de español como segunda lengua. (Tesis de Maestría). Universidad de Costa Rica.

Calsamiglia-Blancafort, H. y Tusón-Valls, A. (2002). Las cosas del decir. Manual de análisis del discurso. Barcelona: Ariel.

Fernández-Leborans, M.J. (1999). El nombre propio. Por Bosque, I. y Demonte, V. (Coord.). Gramática descriptiva de la lengua española. (77-128). Madrid: Espasa.

Halliday M.A.K. (2004). An Introduction to Functional Grammar. Nueva York: Oxford University Press. 
Halliday, M.A.K. y Hasan, R. (1976). Cohesion in English. Nueva York: Longman.

Payne, T.E. (1992). Describing morphosyntax. Cambridge University Press.

Saunders, J.K. (1999). Null and overt references in Spanish second language acquisition: A discourse perspective. (Tesis doctoral). Universidad de Texas en Austin. ProQuest, Dissertations y Theses. (AAT 9947377). [Consulta 1 de noviembre de 2008].

Thornbury, S. (1999). How to teach grammar. England: Pearson Education Limited.

Vargas-Víquez, S. (2012). La adquisición de los recursos gramaticales de referencia por estudiantes de español como segunda lengua en el nivel principiante. Revista de Filología y Lingüística de la Universidad de Costa Rica. 38 (2), 217-238. 